\title{
Single-leg squats can predict leg alignment in dancers performing ballet movements in "turnout"
}

This article was published in the following Dove Press journal:

Open Access Journal of Sports Medicine

16 November 2016

Number of times this article has been viewed

\author{
Luke S Hopper' \\ Nahoko Sato ${ }^{2}$ \\ Andries L Weidemann' \\ 'Western Australian Academy of \\ Performing Arts, Edith Cowan \\ University, Mt Lawley, WA, Australia; \\ ${ }^{2}$ Department of Physical Therapy, \\ Nagoya Gakuin University, Seto, Japan
}

\begin{abstract}
The physical assessments used in dance injury surveillance programs are often adapted from the sports and exercise domain. Bespoke physical assessments may be required for dance, particularly when ballet movements involve "turning out" or external rotation of the legs beyond that typically used in sports. This study evaluated the ability of the traditional single-leg squat to predict the leg alignment of dancers performing ballet movements with turnout. Three-dimensional kinematic data of dancers performing the single-leg squat and five ballet movements were recorded and analyzed. Reduction of the three-dimensional data into a one-dimensional variable incorporating the ankle, knee, and hip joint center positions provided the strongest predictive model between the single-leg squat and the ballet movements. The single-leg squat can predict leg alignment in dancers performing ballet movements, even in "turned out" postures. Clinicians should pay careful attention to observational positioning and rating criteria when assessing dancers performing the single-leg squat.
\end{abstract}

Keywords: injury, motion capture, clinical assessment

\section{Introduction}

Dance injury surveillance programs typically involve applying a battery of physical assessments and prospectively monitoring injury incidence in a dance cohort. These programs aim to identify injury risk factors and ultimately prevent dance injuries. ${ }^{1}$ Physical assessments in dance are often adapted from precedents in sport and exercise. Modern biomechanical analyses have the potential to examine whether the unique movements undertaken by dancers are adequately measured using these precedents from the sport and exercise domain.

Dancing with a valgus leg alignment is cited as a potential risk factor for lower limb injury, the most frequently injured body region in dancers. ${ }^{2-6} \mathrm{~A}$ valgus leg alignment is suggested to cause stress to the ligamentous structures of the knee, and the associated hyper-pronation may cause stress to the medial structures of the foot. ${ }^{7,8}$ In addition, ballet dancers perform with the legs "turned out" or with a combination of active hip and passive knee joint external rotation as well as passive foot pronation. ${ }^{9}$ The torsional loads encountered by the lower limb while dancing in turnout are suggested to increase the likelihood of a dancer moving with a valgus leg alignment. ${ }^{10,11}$ Protocols for assessing dancer leg alignment which incorporate turnout are now being established. ${ }^{12,13}$ The single-leg squat is commonly used as a functional assessment of leg alignment in athletes, it can predict leg alignment during landings and also correlate with hip muscle function. ${ }^{14-19}$ No studies have examined whether the single-leg squat 
can predict dynamic leg alignment during dancing, particularly when a dancer's legs are held in a "turned out" position.

Despite several attempts to develop rating criteria and kinematic parameters to assess the single-leg squat, ${ }^{13,17,20-22}$ evidence for the inter- and intra-rater reliability of the singleleg squat test is inconclusive ${ }^{23}$ and is likely affected by clinician or assessor experience. ${ }^{21,24}$ Subjective assessments of the single-leg squat may be improved with further examination of kinematic parameters that can differentiate performance of the single-leg squat.

The assessment of dancers moving in a "turned out" posture provides challenges for both subjective and quantitative kinematic assessments. The anatomical axis of hip joint which is defined by traditional biomechanical methods ${ }^{25}$ likely changes when a dancer moves with a "turned out" stance. That is, hip flexion with a turned out leg posture would likely be calculated as a combination of hip flexion and abduction. This may affect the ability of standard biomechanical kinematic protocols to assess dancer leg kinematics. ${ }^{12}$ Therefore, kinematic parameters need to be evaluated which can measure dancer leg three-dimensional (3D) kinematics and are not influenced by turnout.

The purpose of this study was to examine the relationship between the leg alignment in dancers performing a single-leg squat test and during the dynamic performance of specific ballet movements. The secondary aim was to develop a kinematic parameter, which can assess dancer leg alignment in both neutral and turnout leg postures. It was hypothesized that the single-leg squat can be used to predict leg alignment during dynamic performances of ballet exercises through the use of a kinematic parameter which is not affected by turned out leg positions.

\section{Methods}

Thirteen injury-free dancers with at least 5 years dance training (male $n=4$, female $n=9$; mean age $19.2 \pm 1.3$ years) enrolled in second or third year of the dance program at the Western Australian Academy of Performing Arts (Edith Cowan University, Australia) participated in this study. The experimental procedure of the study was approved by the Edith Cowan University Human Research Ethics Committee, and written informed consent was obtained from each participant before the commencement of the experiment.

Retro-reflective markers were attached to the dancers' pelvis and lower limbs using the marker placement from Besier et $\mathrm{al}^{26}$ (pelvis: four markers placed on the iliac spines; thigh and shank: three marker clusters on each segment; foot: calcaneous, first and fifth metatarsal heads). Marker trajectory data were captured using 12 Vicon T40S cameras (Oxford Metrics, Oxford, UK) sampling at $250 \mathrm{~Hz}$ while participants performed single-leg squats and five ballet movements. For the single-leg squat, the dancers were instructed to stand in a natural anatomical stance (not parallel, refer Table 1 for turnout angles) and repeat four single-leg squats to their maximum depth. The five ballet movements performed by the dancers were 1) battement fondu with rise (rise), 2) battement fondu with relevé (releve), 3) ballonné en place, 4) ballonné traveling, and 5) sissonne fondue (sissonne) (Video S1). In the rise and relevé, dancers held onto a ballet barre as typical while performing these movements. The order of the five ballet movements described above are considered to be of increasing technical difficulty in ballet. ${ }^{9}$ The dancers repeated four trials of each ballet exercise on both legs in a random order at a standardized tempo of 100 beats per minute. Each repetition of the rise exercise was completed over four beats, the relevé, over two beats and the ballonné exercises in one beat to represent the tempos typically used in dance classes. The single-leg squat and sissonne were not performed to a specific tempo. On completion of the movements, the dancers identified their preferred standing and landing leg for performing the movements.

A mid-pelvis marker was calculated as the average position of the four markers positioned on the dancers' iliac spines. The vertical height of the mid-pelvis marker was used to define the movement phases of each task. All analyses of leg alignment were conducted on the phase in which the vertical position of the dancers' mid-pelvis markers were in lowest $20 \%$ of each repetition, where $0 \%$ represents the lowest

Table I Averaged values of four kinematic parameters during landing phase and maximum value of knee flexion angle during the landing phase

\begin{tabular}{lllllll}
\hline Kinematic parameters & Single-leg squat & Rise & Relevé & Ballonné en place & Ballonné traveling & Sissonne \\
\hline Turnout angle $\left(^{\circ}\right)$ & $11.0 \pm 7.4$ & $67.8 \pm 6.4$ & $59.3 \pm 8.2$ & $49.2 \pm 8.6$ & $44.7 \pm 10.8$ & $82.3 \pm 10.8$ \\
Knee flexion $\left(^{\circ}\right)$ & $67.9 \pm 10.6$ & $41.7 \pm 6.7$ & $40.7 \pm 8.2$ & $49.5 \pm 5.4$ & $48.3 \pm 5.3$ & $48.4 \pm 7.5$ \\
Hip adduction $\left(^{\circ}\right)$ & $-6.7 \pm 8.0$ & $1.0 \pm 4.7$ & $-2.3 \pm 4.4$ & $-1.2 \pm 5.5$ & $-1.9 \pm 5.0$ & $27.4 \pm 6.4$ \\
Thigh tilt $\left(^{\circ}\right)$ & $13.7 \pm 5.9$ & $13.2 \pm 2.1$ & $12.7 \pm 2.2$ & $13.5 \pm 2.7$ & $11.5 \pm 3.2$ & $14.8 \pm 4.9$ \\
Knee-hip distance $(\mathrm{mm})$ & $107.3 \pm 40.0$ & $119.6 \pm 20.2$ & $115.4 \pm 21.3$ & $118.9 \pm 21.2$ & $104.1 \pm 25.0$ & $112.0 \pm 30.3$ \\
\hline
\end{tabular}


vertical position of the mid-pelvis for the repetition and 100\% represents the vertical mid-pelvis height during quiet standing. Three primary variables were analyzed in the assessment of leg alignment during the trials: 1) hip adduction angle, 2) thigh tilt angle, and 3) the horizontal knee-hip distance. The turnout angle of the foot and the peak knee flexion angle were also analyzed as secondary variables. Hip adduction and knee flexion were calculated using standard biomechanical methods for segment kinematic analyses where hip adduction involved the rotation of the thigh segment coordinate system relative to the pelvis and knee flexion involved rotation of the shank coordinate system relative to the thigh. ${ }^{25}$ The pelvis, thigh, shank, and foot coordinate systems were defined as per Besier et al. ${ }^{26}$ Turnout was calculated as a scalar of the long axis of the foot rotated relative to an anteroposterior vector in the coronal plane of the pelvic coordinate system. Thigh tilt and knee-hip distance were calculated using a plane defined by a customized foot coordinate system where the origin was defined at the ankle joint center, the primary axis (Y) was the laboratory vertical axis, the second axis $(Z)$ was the

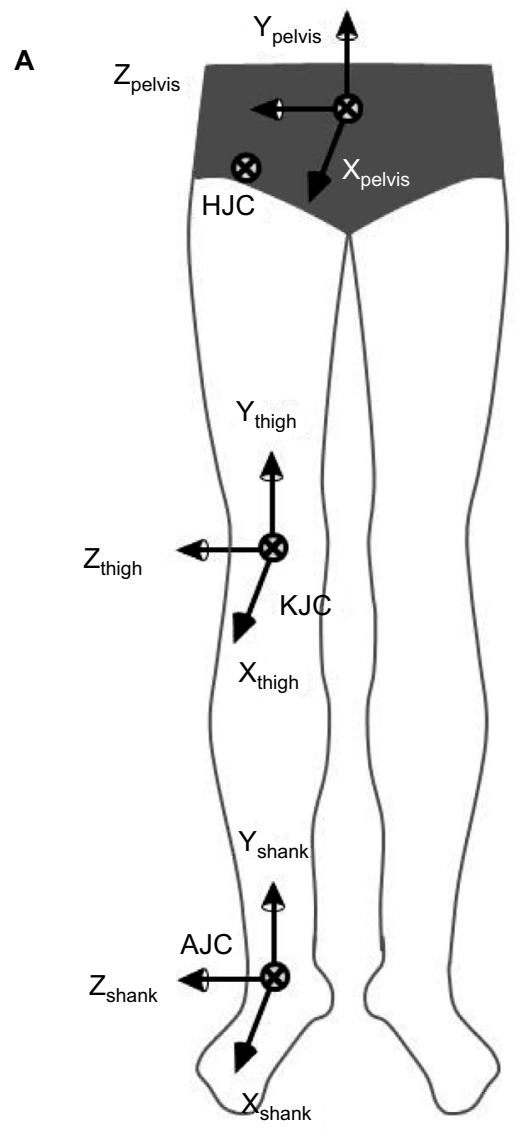

cross product between the primary axis and the longitudinal axis of the traditional foot coordinate system, the third axis (X) was the cross product between the primary and secondary axes. Therefore, the customized foot coordinate system was held at the ankle joint, the Y-axis remained parallel to the laboratory vertical axis, and the $\mathrm{Z}$-axis aligned to the anatomical mediolateral plane of the foot segment for the entire trial (Figure 1) The position of the hip and knee joint centers were then recalculated relative to the customized foot coordinate system. The thigh tilt angle was defined as the angle between the vector $Z Y_{\text {hip }}-Z_{\text {knee }}$ and the $Y$-axis of the customized foot coordinate system in the ZY plane. The knee-hip distance was defined as the distance between $Z_{\text {hip }}$ and $Z_{\text {knee }}$ relative to the customized foot coordinate system.

All variables were compiled as the average of the four respective movement trials for each participant. Two separate analyses of variance with post hoc comparisons were used to compare the peak knee flexion and turnout angles across the single-leg squat and the five ballet movements. Simple linear regression analyses were used to determine whether the mean

B

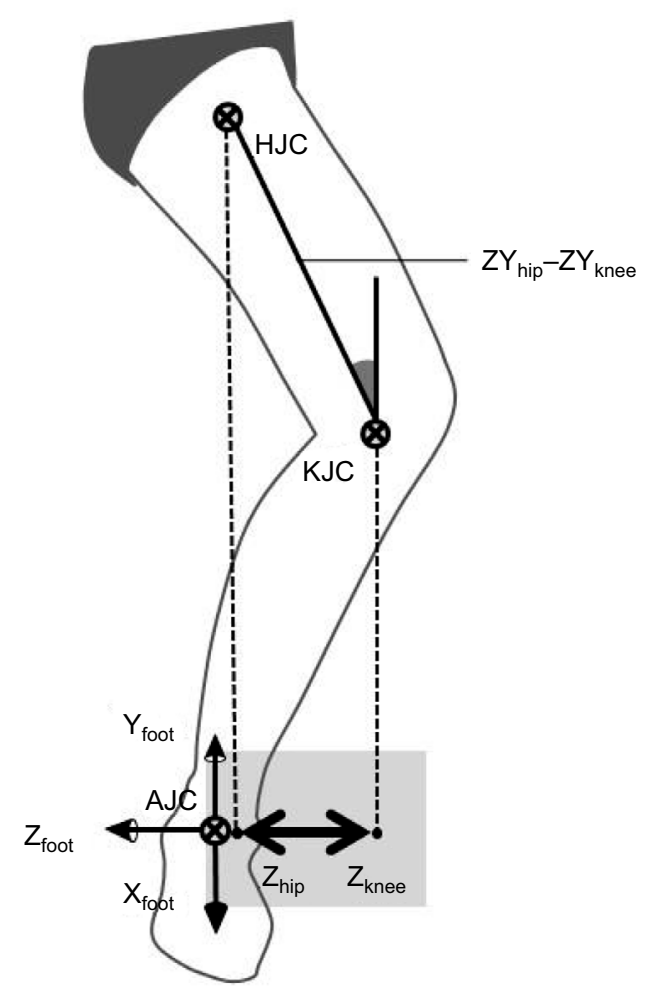

Figure I (A) Anatomical coordinate systems of the pelvis, thigh and shank segments; (B) depiction of the calculation of the thigh tilt and knee-hip distance variables. Notes: (A) Coordinate systems of the pelvis, thigh, and shank segments as well as the hip joint center (HJC), knee joint center (KJC), and ankle joint center (AJC) locations. The pelvis origin was the mid-point of the anterior superior iliac spines of the pelvis, the thigh origin was the KJC and the shank origin was the AJC. Hip adduction was calculated by rotating $X_{\text {thigh }}$ relative to $X_{\text {pelvis }}$. Knee flexion was calculated by rotating $Z_{\text {shank }}$ relative to $Z_{\text {thigh }}$. $(B)$ The customized foot coordinate system held at the $A J C$ and associated calculation of the thigh tilt and knee-hip distance variable. $Z Y_{\text {hip }}$ and $Z Y_{\text {knee }}$ were the $Z$ and $Y$ coordinate values in the customized foot coordinate system of the $\mathrm{HJC}$ and $\mathrm{KJC}$, respectively. The thigh tilt angle was the angle to the vertical of the $Z Y_{\text {hip }}-Z Y_{\text {knee }}$ vector. The knee-hip distance was the scalar distance between $Z_{\text {hip }}$ and $Z_{\text {knee }}$. 
values of the primary variables (hip adduction, thigh tilt angle, and knee-hip distance) of single-leg squat could predict the mean values of the primary variables in the ballet movements (rise, relevé, ballonné en place, ballonné traveling, and sissonne) for the lowest $20 \%$ phase of the movements. Pearson correlation coefficients were calculated to examine the relationship between the primary variables and the turnout and knee flexion angles. All statistical analyses were performed using SPSS Version 23.0 (IBM Corporation, Armonk, NY, USA) with statistical significance set at $p<0.05$.

\section{Results}

Table 1 provides the averaged values for the turnout, knee flexion, hip adduction, thigh tilt angles, and knee-hip distance for the low $20 \%$ pelvis phase for all exercises. There was a significant effect for the turnout angle $[F(5,60)=160.9$, $p<0.001]$ and turnout was significantly different $(p<0.01)$ between all ballet movements except for ballonné en place and ballonné traveling. There was also a significant effect for the knee flexion angle $[\mathrm{F}(3,38)=36.7, p<0.001]$, and the single-leg squat was significantly larger $(p<0.01)$ than all ballet movements. There were also significant differences between the peak knee flexion angles for ballet movements; rise $>$ ballonné en place $(p<0.01)$, rise $>$ ballonné traveling $(p<0.05)$, and relevé $>$ ballonné en place $(p<0.01)$.
Simple linear regression analyses revealed that the knee-hip distance for the single-leg squat was a significant predictor $(p<0.05)$ of the knee-hip distance for all ballet movements and the thigh tilt angle for the single-leg squat was a significant predictor of the thigh tilt angle for relevé, ballonné en place, ballonné traveling, and sissonne. The beta values of the knee-hip distance were higher than that of the thigh tilt angle for all respective regressions. The hip adduction angle during the single-leg squat did not predict the hip adduction for any of the ballet movements (Figure 2).

No significant correlations were observed between turnout and the knee-hip distances or thigh tilt angles (Table 2), However, the hip adduction angle of the sissonne movement was moderately correlated with the turnout angle $(r=0.571$, $p<0.05)$. There was no significant correlation between the knee flexion angle and the knee-hip distance or hip adduction angle, whereas the thigh tilt angle for rise and sissonne were moderately correlated with the knee flexion angle ( $r=0.614$, $p<0.05 ; r=0.574, p<0.05$, respectively; Table 2 ).

\section{Discussion}

Kinematic parameter, the knee-hip distance when observed in dancers performing single-leg squats, predicted dancer leg alignment during dynamic ballet movements. This finding supports the presumption that the leg alignment during single-leg squats can predict the leg alignment dancers exhibit

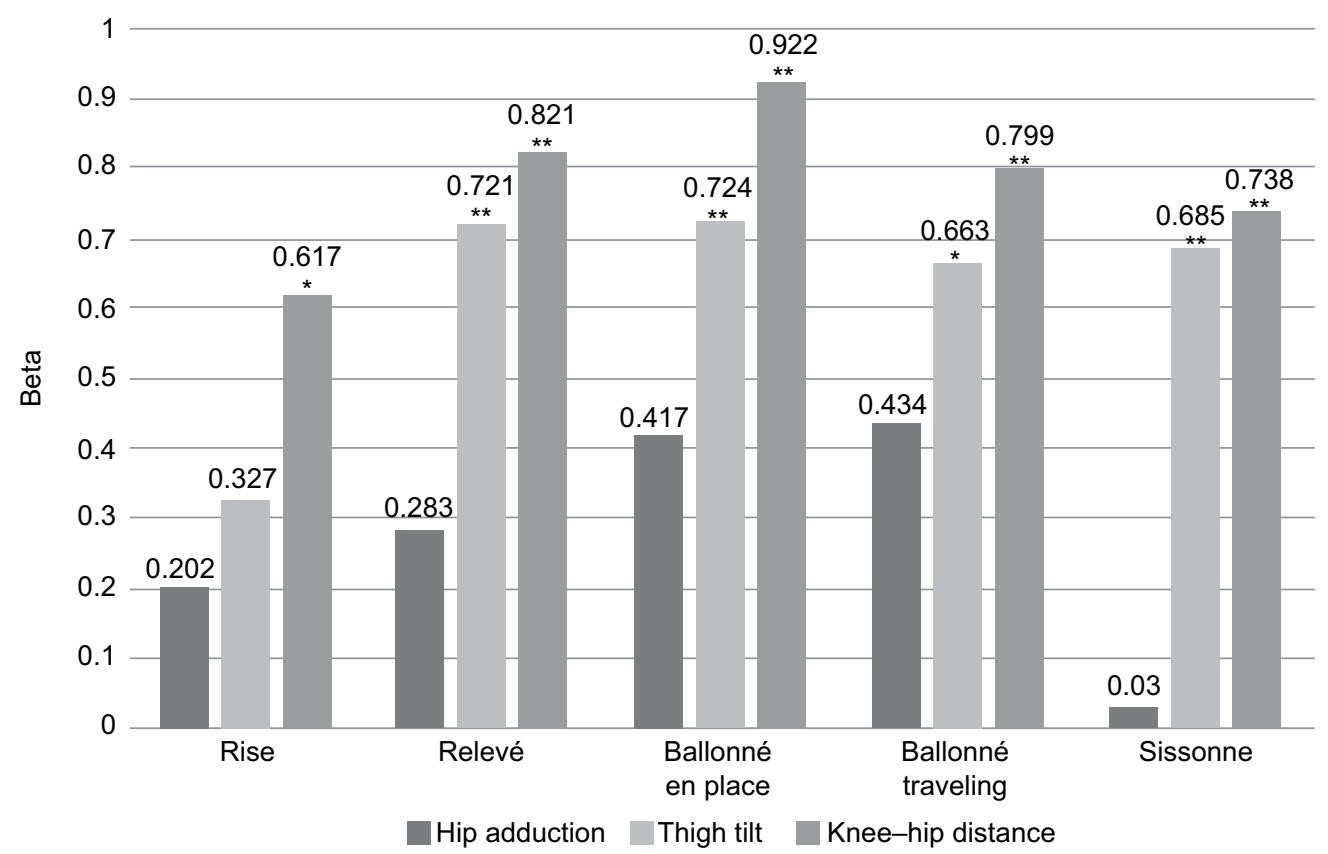

Figure 2 Beta values from the linear regressions of the kinematic parameters (hip adduction, thigh tilt, and knee-hip distance) from the single-leg squat as predictors of the respective variable in the ballet movement. ${ }^{*} p<0.05, * * p<0.01$. 
Table 2 Correlations between the kinematic parameters and the turnout and knee flexion angles

\begin{tabular}{|c|c|c|c|c|c|c|}
\hline Kinematic parameters & & Rise & Relevé & Ballonné en place & Ballonné traveling & Sissonne \\
\hline \multirow[t]{3}{*}{ Turnout } & Hip adduction & 0.540 & 0.405 & 0.461 & 0.291 & $0.57 I^{*}$ \\
\hline & Thigh tilt & 0.015 & 0.172 & 0.331 & 0.522 & 0.532 \\
\hline & $\mathrm{KJC}-\mathrm{HJC}$ & -0.243 & -0.115 & -0.090 & 0.250 & 0.533 \\
\hline \multirow[t]{3}{*}{ Knee flexion } & Hip adduction & 0.288 & 0.242 & 0.155 & -0.054 & -0.068 \\
\hline & Thigh tilt & $0.614^{*}$ & 0.420 & 0.492 & 0.410 & $0.574^{*}$ \\
\hline & $\mathrm{KJC}-\mathrm{HJC}$ & 0.403 & 0.475 & 0.424 & 0.495 & 0.476 \\
\hline
\end{tabular}

Note: $*_{p}<0.05$.

Abbreviations: KJC, knee joint center; HJC, hip joint center.

during "turned out" ballet movements. This study also supports previous reports of the positive relationship between single-leg squats and leg alignment during landings in athletic populations. ${ }^{14,15}$ These findings warrant the use of the singleleg squat in prospective dance injury surveillance etiological studies as a means to assess dancer functional leg alignment.

The knee-hip distance parameter was a stronger predictor of leg alignment than the hip adduction and the thigh tilt angle during the ballet movements in this study. The poor predictive ability of the hip adduction variable is likely due to cross talk between the hip flexion and adduction as a result of the external rotation or turnout of the hips during the ballet movements as well as confounding motion of the pelvis such as in the sissonne involving hip extension. The thigh tilt angle uses a two-dimensional (2D) planar vector. The vertical length of the thigh vector reduces toward zero as the hip joint lowers toward the vertical height of the knee during knee flexion. This changing vector length will influence the resultant thigh tilt angle, and therefore, the measure is likely confounded by the amount of knee flexion demonstrated in each trial. The knee-hip distance only considered the horizontal components of the knee and hip joint centers relative to the customized foot coordinate system thereby removing the influence of both turnout (through the use of the customized foot coordinate system) and knee flexion (by removing the vertical components of the joint center positions). The reductionist approach of the knee-hip distance appears to be a conducive means of assessing leg alignment in the dancers.

The reliability of subjective clinical assessments of leg alignment during single-leg squats has received conflicting reports. ${ }^{17,20-22}$ The results of the present study suggest that clinicians should carefully consider the perspective in which leg alignment is observed and the means in which leg alignment is assessed during single-leg squats. Clinical evaluation of the single-leg squat may benefit from the clinician standing in line with the long axis of the foot ${ }^{13}$ and assess leg alignment as the horizontal distance between the hip and the knee and not as the angle of the thigh relative to the vertical. The 2D perspective of clinicians observing the single-leg squat will encounter the same perspective error associated with knee flexion in assessing thigh tilt which was encountered in the present analyses. Assessing leg alignment through subjective analysis of our knee-hip distance measure may result in higher reliability in clinical assessments of the single-leg squat for use in dance injury surveillance programs.

This is the first study to quantify turnout positions used by dancers across a diverse range of ballet movements. Assessment of the turnout capacities in dancers has been the focus of many research studies which have predominantly focused on the relationship between turnout in a standing position and the active and passive hip joint external rotation ranges of motion. ${ }^{10,27-30}$ The results of the present study reported turnout angles during rise and sissonne to be greater than those reported in previous research studies. ${ }^{10,27,28,30}$ However, the turnout angle of sissonne is likely overestimated due to the oblique anteriorly tilted position of the pelvis during the task that results during from the hip extension of the opposite leg. Nonetheless, this preliminary data may be of benefit for clinical assessments of dancer turnout and as return to function measures for rehabilitation.

\section{Conclusion}

The single-leg squat can provide a robust functional assessment of dancers as it predicted dancer leg alignment during various ballet movements which involve "turnout" of the legs. This predictive relationship was only observed when 3D leg alignment data were reduced to a one-dimensional variable calculated in a customized coordinate system which represented a frontal plane through the foot. Clinical assessments of single-leg squat performance should carefully consider observational positions and assessment measures for the single-leg squat.

\section{Acknowledgment}

This work was in part produced during a sabbatical research fellowship supported by Nagoya Gakuin University. 


\section{Disclosure}

The authors report no conflicts of interest in this work.

\section{References}

1. Liederbach M, Hagins M, Gamboa JM, Welsh TM. Assessing and reporting dancer capacities, risk factors, and injuries: recommendations from the IADMS standard measures consensus initiative. J Dance Med Sci. 2012;16(4):139-153.

2. Russell JA. Preventing dance injuries: current perspectives. Open Access J Sports Med. 2013;4:199-210.

3. Gamboa JM, Roberts LA, Maring J, Fergus A. Injury patterns in elite preprofessional ballet dancers and the utility of screening programs to identify risk characteristics. J Orthop Sports Phys Ther. 2008;38(3):126-136.

4. Jacobs CL, Hincapié CA, Cassidy JD. Musculoskeletal injuries and pain in dancers: a systematic review update. J Dance Med Sci. 2012;16(2):74-84.

5. Bowerman E, Whatman C, Harris N, Bradshaw E. A review of the risk factors for lower extremity overuse injuries in young elite female ballet dancers. J Dance Med Sci. 2015;19(2):51-56.

6. Bowerman E, Whatman C, Harris N, Bradshaw E, Karin J. Are maturation, growth and lower extremity alignment associated with overuse injury in elite adolescent ballet dancers? Phys Ther Sport. 2014;15(4):234-241.

7. Howse J, McCormack M. Anatomy, Dance Technique and Injury Prevention. London: A\&C Black; 2009.

8. Fitt SS. Dance kinesiology. Vol. 3. New York: Schirmer; 1996.

9. Royal Academy of Dance. The Foundations of Classical Ballet Technique. 1st ed. London: Royal Academy of Dance; 2008.

10. Coplan JA. Ballet dancer's turnout and its relationship to self-reported injury. J Orthop Sports Phys Ther. 2002;32(11):579-584.

11. Bowerman EA, Whatman C, Harris N, Bradshaw E. A review of the risk factors for lower extremity overuse injuries in young elite female ballet dancers. J Dance Med Sci. 2015;19(2):51-56.

12. Gontijo KNS, Candotti CT, dos Santos Feijó G, Ribeiro LP, Loss JF. Dynamic evaluation method of lower limbs joint alignment (MADAAMI) for dancers during the plié. Rev Bras Ciênc Esporte. In press 2016.

13. Bowerman E, Whatman C, Harris N, Bradshaw E. Reliability of 2D lower extremity alignment measures in elite adolescent ballet dancers. N Z J Sports Med. 2013;40(2):70-73.

14. Stensrud S, Myklebust G, Kristianslund E, Bahr R, Krosshaug T. Correlation between two-dimensional video analysis and subjective assessment in evaluating knee control among elite female team handball players. Br J Sports Med. 2011;45(7):589-595.

15. Ugalde V, Brockman C, Bailowitz Z, Pollard CD. Single leg squat test and its relationship to dynamic knee valgus and injury risk screening. PM\&R. 2015;7(3):229-235.
16. Claiborne TL, Armstrong CW, Gandhi V, Pincivero DM. Relationship between hip and knee strength and knee valgus during a single leg squat. J Appl Biomech. 2006;22(1):41-50.

17. Crossley KM, Zhang WJ, Schache AG, Bryant A, Cowan SM. Performance on the single-leg squat task indicates hip abductor muscle function. Am J Sports Med. 2011;39(4):866-873.

18. Nguyen A-D, Shultz SJ, Schmitz RJ, Luecht RM, Perrin DH. A preliminary multifactorial approach describing the relationships among lower extremity alignment, hip muscle activation, and lower extremity joint excursion. J Athl Train. 2011;46(3):246-256.

19. Willson JD, Ireland ML, Davis I. Core strength and lower extremity alignment during single leg squats. Med Sci Sports Exerc. 2006;38(5):945-952.

20. Ageberg E, Bennell KL, Hunt MA, Simic M, Roos EM, Creaby MW. Validity and inter-rater reliability of medio-lateral knee motion observed during a single-limb mini squat. BMC Musculoskelet Disord. 2010;11(1):1-8.

21. Whatman C, Hume P, Hing W. The reliability and validity of physiotherapist visual rating of dynamic pelvis and knee alignment in young athletes. Phys Ther Sport. 2013;14(3):168-174.

22. Chmielewski TL, Hodges MJ, Horodyski M, Bishop MD, Conrad BP, Tillman SM. Investigation of clinician agreement in evaluating movement quality during unilateral lower extremity functional tasks: a comparison of 2 rating methods. J Orthop Sports Phys Ther. 2007;37(3):122-129.

23. McCunn R, aus der Fünten K, Fullagar HHK, McKeown I, Meyer T. Reliability and association with injury of movement screens: a critical review. Sports Med. 2016;46(6):763-781.

24. Weeks BK, Carty CP, Horan SA. Kinematic predictors of single-leg squat performance: a comparison of experienced physiotherapists and student physiotherapists. BMC Musculoskelet Disord. 2012;13(1):1-7.

25. Wu G, Siegler S, Allard P, et al. ISB recommendation on definitions of joint coordinate system of various joints for the reporting of human joint motion - part I: ankle, hip, and spine. J Biomech. 2002;35(4):543-548.

26. Besier TF, Sturnieks DL, Alderson JA, Lloyd DG. Repeatability of gait data using a functional hip joint centre and a mean helical knee axis. J Biomech. 2003;36(8):1159-1168.

27. van Merkensteijn GG, Quin E. Assessment of compensated turnout characteristics and their relationship to injuries in university level modern dancers. J Dance Med Sci. 2015;19(2):57-62.

28. Sutton-Traina K, Smith JA, Jarvis DN, Lee S-P, Kulig K. Exploring active and passive contributors to turnout in dancers and non-dancers. Med Probl Perform Art. 2015;30(2):78-83.

29. Negus V, Hopper D, Briffa NK. Associations between turnout and lower extremity injuries in classical ballet dancers. J Orthop Sports Phys Ther. 2005;35(5):307-318.

30. Welsh TM, Rodriguez M, Beare LW, Barton B, Judge T. Assessing turnout in university dancers. J Dance Med Sci. 2008;12(4):136-141.
Open Access Journal of Sports Medicine

\section{Publish your work in this journal}

The Open Access Journal of Sports Medicine is an international, peer-reviewed, open access journal publishing original research, reports, reviews and commentaries on all areas of sports medicine. The journal is included on PubMed. The manuscript management system is completely online and includes a very quick and fair
Dovepress

peer-review system. Visit http://www.dovepress.com/testimonials.php to read real quotes from published authors. 